\title{
Decomposition of Involutions on Inertially Split Division Algebras
}

\author{
Patrick J. Morandi B.A. Sethuraman
}

December 22, 2000

\begin{abstract}
Let $F$ be a Henselian valued field with $\operatorname{char}(\bar{F}) \neq 2$, and let $S$ be an inertially split $F$-central division algebra with involution $\sigma^{*}$ that is trivial on an inertial lift in $S$ of the field $Z(\bar{S})$. We prove necessary and sufficient conditions for $S$ to contain a $\sigma^{*}$ stable quaternion $F$-subalgebra, and for $\left(S, \sigma^{*}\right)$ to decompose into a tensor product of quaternion algebras. These conditions are in terms of decomposability of an associated residue central simple algebra $\bar{I}$ that arises from a Brauer group decomposition of $S$.
\end{abstract}

\section{Introduction}

Let $S$ be a central simple algebra with center $F$. If $S$ has an involution $\sigma$, then a fundamental problem is to determine when does $(S, \sigma)$ decompose. In other words, when are there proper $F$-subalgebras $S_{1}$ and $S_{2}$ such that $S=S_{1} \otimes_{F} S_{2}$ and $\sigma=\sigma_{1} \otimes \sigma_{2}$ for some involutions $\sigma_{i}$ on $S_{i}$ ? If $\operatorname{deg}(S)=4$ and $\left.\sigma\right|_{F}=$ id, then a theorem of Albert [?, Ch. XI, Thm. 9] says that $S$ decomposes into a tensor product of quaternion algebras. However, it may not be the case that $(S, \sigma)$ decomposes; the first example of an indecomposable involution was given by Amitsur, Rowen, and Tignol in [?]. In [?], Knus, Parimala, and Sridharan gave a necessary and sufficient condition on an algebra of degree 4 with involution (of the first kind) to decompose. For algebras with involution of larger degree, it is generally a difficult question to determine when it decomposes.

In [?] Dherte studied decomposability of involutions on certain Malcev-Neumann division algebras. More precisely, if $K / F$ is an elementary Abelian 2-extension with Galois group $G$, and if $f \in Z^{2}\left(G, K^{*}\right)$, then one can construct the Malcev-Neumann series division ring $S(A / K / F)$, where $A$ is the crossed product algebra $(K / F, G, f)$. The center $\widehat{F}$ of $S(A / K / F)$ is a field of formal series over $F$, and $S(A / K / F)$ has a maximal subfield $\widehat{K}$ such that $\operatorname{Gal}(\widehat{K} / \widehat{F})=G$. Moreover, $S(A / K / F)=(\widehat{K} / \widehat{F}, G, \widehat{f})$ for a cocycle $\widehat{f}$ related to $f$. If $S(A / K / F)=\oplus_{g \in G} \widehat{K} z_{g}$ with $z_{g} z_{h}=\widehat{f}(g, h) z_{g h}$ and $A=\oplus_{g \in G} K x_{g}$ with $x_{g} x_{h}=f(g, h) x_{g h}$, then there is an involution $\sigma^{*}$ on $S(A / K / F)$ with $\left.\sigma^{*}\right|_{\widehat{K}}=$ id and $\sigma^{*}\left(z_{g}\right)=z_{g}$, and an involution $\sigma$ on $A$ with $\left.\sigma\right|_{K}=$ id and $\sigma\left(x_{g}\right)=x_{g}$. Dherte proved that $S(A / K / F)$ has a 
$\sigma^{*}$-stable $\widehat{F}$-central quaternion algebra if and only if $A$ has a $\sigma$-stable $F$-central quaternion algebra, and also that $\left(S(A / K / F), \sigma^{*}\right)$ decomposes into a tensor product of quaternion algebras if and only if $(A, \sigma)$ decomposes into a tensor product of quaternion algebras. A fundamental technique in [?] was valuation theory; the field $\widehat{F}$ has a Henselian valuation with residue field $F$, and so this valuation extends to $S(A / K / F)$.

In this paper we study the question of decomposability of involutions on inertially split division algebras defined over Henselian valued fields. To be more precise, let $F$ be a Henselian valued field with $\operatorname{char}(\bar{F}) \neq 2$. If $S$ is an $F$-central division algebra, then $S$ is said to be inertially split if there is an inertial extension $K / F$ such that $K$ is a splitting field for $S$. The structure of inertially split division algebras was investigated by Jacob and Wadsworth in [?, Sec. 5]. In particular, by [?, Lemma 5.14], there is a natural but not unique decomposition of $S$, up to similarity, as $S \sim I \otimes_{F} N$, where $I$ is similar to an inertial division algebra and $N$ is a nicely semiramified division algebra (see [?, Sec. 2, 3] for definitions). For the Malcev-Neumann algebra $S(A / K / F)$ mentioned above, by viewing $f \in Z^{2}\left(G, \widehat{K}^{*}\right)$, the cocycle $\widehat{f}$ factors as $\widehat{f}=f c$ with $c$ a symmetric cocycle having values in $\widehat{F}^{*}$, and this factorization gives a similarity relation $S(A / K / F) \sim\left(A \otimes_{F} \widehat{F}\right) \otimes_{\widehat{F}}(\widehat{K} / \widehat{F}, G, c)$, which is a special case of the decomposition of [?]. The algebra $A$, if it is a division algebra, is the residue algebra of the inertial algebra $A \otimes_{F} \widehat{F}$.

Suppose that $S \sim I \otimes_{F} N$ as above has exponent 2. By starting with an involution $\sigma^{*}$ on $S$ that is trivial on an inertial lift of $Z(\bar{S})$, and by representing $S$ and $I$ as generalized crossed products in a natural way, we produce an involution $\sigma$ on $I$, and $\sigma$ induces an involution $\bar{\sigma}$ on an $\bar{F}$-central simple algebra $\bar{I}$. The algebra $\bar{I}$ is, in some sense, the residue algebra of the central simple algebra $I$. We prove that $S$ has an $F$-central quaternion subalgebra stable under $\sigma^{*}$ if and only if $\bar{I}$ has an $\bar{F}$-central quaternion subalgebra stable under $\bar{\sigma}$, and $\left(S, \sigma^{*}\right)$ decomposes into a tensor product of quaternion algebras if and only if $(\bar{I}, \bar{\sigma})$ decomposes into a tensor product of quaternion algebras. We thus extend Dherte's results to the context of inertially split algebras over a Henselian valued field with residue characteristic not 2 . We remark that in [?, Thm. 5] it is proved that if $D$ is a division algebra of exponent 2 over a Henselian valued field $F$ (with $\operatorname{char}(\bar{F}) \neq 2$ ) and if $D$ has an involution $\sigma$ of the first kind with $\bar{\sigma}=$ id on $\bar{D}$, then $D$ can be decomposed as $D=S \otimes_{F} T$ with $S$ inertially split and $T$ totally ramified, and each of $S$ and $T$ are stable under $\sigma$. Moreover, $\left(T,\left.\sigma\right|_{T}\right)$ decomposes into a tensor product of quaternion algebras. Thus, at least for involutions with $\bar{\sigma}=\mathrm{id}$, the question of decomposability reduces to the case of inertially split division algebras.

If $F$ is a field with Henselian valuation $v$, recall (e.g., [?, Thm.]) that $v$ extends uniquely to a valuation on any finite dimensional $F$-division algebra. If $D$ is an $F$-central division algebra with valuation, we will denote the value group of $D$ by $\Gamma_{D}$ and the residue division algebra by $\bar{D}$. The group of valuation units of $D$ will be denoted $U_{D}$, and the subgroup of 1 -units by $U_{1, D}$. If $d$ is in the valuation ring of $D$, we denote the image of $d$ in $\bar{D}$ by $\bar{d}$. Recall that the valuation ring is stable under $F$-algebra automorphisms of $D$ and under involutions of the first kind; this is a consequence of [?, Thm.]. Therefore, an F-algebra automorphism $\varphi$ (resp. involution) of $D$ induces an automorphism $\bar{\varphi}$ (resp. involution) of $\bar{D}$ by $\bar{\varphi}(\bar{d})=\overline{\varphi(d)}$. 
We give some more notation that we will use throughout this paper. If $F$ is a field, then $\operatorname{Br}(F)$ is the Brauer group of classes of central simple $F$-algebras, and $\mathrm{Br}_{2}(F)$ is the 2-torsion subgroup of $\operatorname{Br}(F)$. If $S$ is a central simple algebra and $B$ is a subalgebra of $S$, then $C_{S}(B)$ is the centralizer of $B$ in $S$. For $s \in S^{*}$, the inner automorphism $x \mapsto s x s^{-1}$ will be denoted by $\operatorname{Int}(x)$, and the group of $F$-algebra automorphisms of $S$ by $\operatorname{Aut}_{F}(S)$. The reduced norm on $S$ will be denoted by $\mathrm{Nrd}$, or by $\operatorname{Nrd}_{S}$ if we are working with more than one central simple algebra.

If $S$ is an $F$-central simple algebra, then an involution $\sigma$ on $S$ is an anti-automorphism of $S$ with $\sigma^{2}=$ id. If $\left.\sigma\right|_{F}=$ id, then $\sigma$ is said to be of the first kind; otherwise $\sigma$ is of the second kind. We will write $\operatorname{Sym}(S, \sigma)$ and $\operatorname{Skew}(S, \sigma)$ to denote the $F$-subspaces of symmetric and skew-symmetric elements of $\sigma$, respectively. If $\sigma$ is of the first kind and if $n=\operatorname{deg}(S)$, then it is known that $\operatorname{dim}_{F}(\operatorname{Sym}(S, \sigma))=\frac{1}{2} n(n+\varepsilon)$ with $\varepsilon$ either 1 or -1 . We refer to $\varepsilon$ as the type of $\sigma$. Involutions of type 1 are often called orthogonal, and symplectic if they are of type -1 . If $\sigma$ is of the second kind and if $F_{0}$ is the fixed field of $F$ under $\sigma$, then $\operatorname{Sym}(S, \sigma)$ is an $F_{0}$-subspace of $S$, and $\operatorname{dim}_{F_{0}}(\operatorname{Sym}(S, \sigma))=\frac{1}{2} \operatorname{dim}_{F_{0}}(S)$.

In this paper we will use generalized crossed products, which we now describe briefly. More details and proofs about generalized cross products can be found in [?] or [?]. If $Z / F$ is a finite Galois extension with $G=\operatorname{Gal}(Z / F)$ and if $C$ is a $Z$-central simple algebra, then a generalized cocycle of $G$ with values in $C^{*}$ is a pair $(\omega, f)$ of maps with

$$
\begin{gathered}
\omega: G \rightarrow \operatorname{Aut}_{Z}(C), \\
f: G \times G \rightarrow C^{*}
\end{gathered}
$$

such that $\left.\omega_{g}\right|_{Z}=g$ for $g \in G$, and

$$
\begin{aligned}
\omega_{g} \circ \omega_{h} & =\operatorname{Int}(f(g, h)) \circ \omega_{g h}, \\
f(g, h) f(g h, k) & =\omega_{g}(f(h, k)) f(g, h k)
\end{aligned}
$$

for all $g, h, k \in G$. We will refer to the set of generalized cocycles of $G$ with values in $C^{*}$ by $\mathcal{Z}\left(G, C^{*}\right)$.

Given a generalized cocycle $(\omega, f) \in \mathcal{Z}\left(G, C^{*}\right)$, we can construct the generalized crossed product $(C, G,(\omega, f))=\oplus_{g \in G} C x_{g}$, where multiplication is determined by

$$
\begin{aligned}
x_{g} c & =\omega_{g}(c) x_{g}, \\
x_{g} x_{h} & =f(g, h) x_{g h}
\end{aligned}
$$

for all $c \in C$ and $g, h \in G$. This is an $F$-central simple algebra containing $C$, and the centralizer of $Z$ in $(C, G,(\omega, f))$ is precisely $C$. We will call the $x_{g}$ cocycle generators for $(C, G,(\omega, f))$.

Conversely, if $S$ is an $F$-central algebra containing $Z$, then set $C=C_{S}(Z)$. We can write $S$ as a generalized crossed product in the following way. By the Noether-Skolem theorem, 
for each $g \in G$ there is an $x_{g} \in S^{*}$ with $\left.\operatorname{Int}\left(x_{g}\right)\right|_{Z}=g$. Let $\omega: G \rightarrow \operatorname{Aut}_{F}(C)$ be given by $\omega_{g}=\left.\operatorname{Int}\left(x_{g}\right)\right|_{C}$, and define $f: G \times G \rightarrow C^{*}$ by $f(g, h)=x_{g} x_{h} x_{g h}^{-1}$. A short calculation shows that $(\omega, f) \in \mathcal{Z}\left(G, C^{*}\right)$ and $S=\oplus_{g \in G} C x_{g}$, so $S=(C, G,(\omega, f))$.

If $(\omega, f)$ and $\left(\omega^{\prime}, f^{\prime}\right)$ are two generalized cocycles of $G$ with values in $C^{*}$, then they are said to be cohomologous if there are elements $c_{g} \in C^{*}$ such that

$$
\begin{aligned}
\omega_{g}^{\prime} & =\operatorname{Int}\left(c_{g}\right) \circ \omega_{g}, \\
f^{\prime}(g, h) & =c_{g} \omega_{g}\left(c_{h}\right) f(g, h) c_{g h}^{-1}
\end{aligned}
$$

for all $g, h \in G$. The $F$-algebras $(C, G,(\omega, f))$ and $\left(C, G,\left(\omega^{\prime}, f^{\prime}\right)\right)$ are isomorphic if and only if $(\omega, f)$ and $\left(\omega^{\prime}, f^{\prime}\right)$ are cohomologous.

\section{Involutions on Generalized Crossed Products}

In this section we investigate involutions on generalized crossed products. As pointed out in the introduction, if $S$ is an inertially split $F$-central division algebra, we will decompose $S \sim I \otimes_{F} N$, and we will represent $S$ and $I$ as generalized crossed products, each of the form $(C, G,(\omega, f))$ with $C$ a division algebra and $Z(C)$ an elementary Abelian 2-extension of $F$ with Galois group $G$. We will use the results of this section to associate to an involution on $S$ an involution on a residue algebra $\bar{I}$, and to write $S, I$, and $\bar{I}$ as generalized crossed products in a convenient way with regard to the involutions.

We will use the following lemma to show that if $S$ has an involution of the first kind, then $S$ has an involution that is the identity on the subfield $Z(C)$.

Lemma 2.1 Let $T$ be an F-central simple algebra of exponent 2. Suppose that $Z$ is a subfield of $T$ that contains $F$ such that $C_{T}(Z)$ is a division algebra. Then there is an involution $\sigma$ on $T$ with $\left.\sigma\right|_{Z}=\mathrm{id}$. Moreover, if $\sigma$ is an involution on $C_{T}(Z)$ that is trivial on $Z$, then $\sigma$ extends to an involution on $T$.

Proof. Since $2[T]=0$ in $\operatorname{Br}(F)$, there is an involution $\tau$ of the first kind on $T$ by $[$ ?, $\S 16$, Thm. 1]. The field $\tau(Z)$ is $F$-isomorphic to $Z$, so there is an $x \in T^{*}$ with $x a x^{-1}=\tau(a)$ for all $a \in Z$. Applying $\tau$ to the equation $x a=\tau(a) x$, we get $\tau(a) \tau(x)=\tau(x) a$. If $\varepsilon= \pm 1$, then

$$
x a x^{-1}(\tau(x)+\varepsilon x)=\tau(a)(\tau(x)+\varepsilon x)=(\tau(x)+\varepsilon x) a .
$$

Multiplying by $x^{-1}$ on the left gives

$$
a x^{-1}(\tau(x)+\varepsilon x)=x^{-1}(\tau(x)+\varepsilon x) a
$$

which implies that $x^{-1}(\tau(x)+\varepsilon x) \in C_{T}(Z)$, or $\tau(x)+\varepsilon x \in x C_{T}(Z)$. Since both $\tau(x)+x$ and $\tau(x)-x$ cannot be zero, one is invertible as $C_{T}(Z)$ is a division algebra. By conjugating $\sigma$ by whichever is nonzero, we obtain an involution on $T$ that is the identity on $Z$. 
Now, suppose that $\sigma$ is an involution of the first kind on $C_{T}(Z)$. Since $2[T]=0$, there is an involution $\tau$ on $T$ with $\left.\tau\right|_{Z}=$ id by the first paragraph. Then $\tau\left(C_{T}(Z)\right)=C_{T}(Z)$, so $\tau$ restricts to an involution of the first kind on $C_{T}(Z)$. Therefore, there is an $x \in C_{T}(Z)$ with $\sigma(x)= \pm x$ and $\left.\tau\right|_{C_{T}(Z)}=\operatorname{Int}(x) \circ \sigma$. Then $\tau(x)= \pm x$, and $\operatorname{so} \operatorname{Int}\left(x^{-1}\right) \circ \tau$ is an involution on $T$ that extends $\sigma$.

Let $Z / F$ be an elementary Abelian 2-extension with Galois group $G$. If $C$ is a $Z$-central division algebra, we show that if $T=(C, G,(\omega, f))$ is a generalized crossed product that has an involution $\sigma$ with $\left.\sigma\right|_{Z}=$ id, then we may find new cocycle generators to represent $T=\oplus_{g} C x_{g}$ with $\sigma\left(x_{g}\right)=x_{g}$. Note that the previous lemma shows that $T$ has an involution $\sigma$ with $\left.\sigma\right|_{Z}=$ id provided that $[T] \in \mathrm{Br}_{2}(F)$.

Proposition 2.2 Let $C / Z$ be a division algebra and let $T=(C, G,(\omega, f))=\oplus_{g} C x_{g}$ be a generalized crossed product. Suppose that $\sigma$ is an involution on $T$ with $\left.\sigma\right|_{Z}=\mathrm{id}$. Then the generalized cocycle $(\omega, f)$ can be replaced by a cohomologous cocycle $\left(\omega^{\prime}, f^{\prime}\right)$ in such a way that $\sigma$ can be extended to $T=\left(C, G,\left(\omega^{\prime}, f^{\prime}\right)\right)=\oplus_{g} D x_{g}^{\prime}$ with $\sigma\left(x_{g}^{\prime}\right)=x_{g}^{\prime}$.

Proof. We note a few things that we will use in the proof. First, let $\lambda_{g}=\omega_{g} \circ \sigma$, an antiautomorphism of $C$. The map $\lambda_{g}^{2}$ is an automorphism that fixes $Z$. Moreover, if $c_{g}=\sigma\left(x_{g}\right) x_{g}^{-1}$, then we claim that $c_{g} \in C^{*}$ and $\lambda_{g}^{2}=\left.\operatorname{Int}\left(c_{g}^{-1}\right)\right|_{C}$. To see that $c_{g} \in C$, we must show that it commutes with $Z$. If $a \in Z$, then

$$
\begin{aligned}
\sigma\left(x_{g}\right) x_{g}^{-1} a & =\sigma\left(x_{g}\right) g(a) x_{g}^{-1}=\sigma\left(g(a) x_{g}\right) x_{g}^{-1} \\
& =\sigma\left(x_{g} a\right) x_{g}^{-1}=a \sigma\left(x_{g}\right) x_{g}^{-1} .
\end{aligned}
$$

This proves that $c_{g} \in C^{*}$. Next, we have

$$
\begin{aligned}
\lambda_{g}^{2} & =\omega_{g} \circ \sigma \circ \omega_{g} \circ \sigma=\left.\left.\operatorname{Int}\left(x_{g}\right)\right|_{C} \circ \sigma \circ \operatorname{Int}\left(x_{g}\right)\right|_{C} \circ \sigma \\
& =\left.\left.\operatorname{Int}\left(x_{g}\right)\right|_{C} \circ \operatorname{Int}\left(\sigma\left(x_{g}\right)^{-1}\right)\right|_{C} \circ \sigma^{2}=\left.\operatorname{Int}\left(x_{g} \sigma\left(x_{g}\right)^{-1}\right)\right|_{C}=\left.\operatorname{Int}\left(c_{g}^{-1}\right)\right|_{C},
\end{aligned}
$$

which gives the second claim. Furthermore, since $\sigma\left(x_{g}\right)=c_{g} x_{g}$, applying $\sigma$ to this equation yields $x_{g}=c_{g} x_{g} \sigma\left(c_{g}\right)=c_{g} \lambda_{g}\left(c_{g}\right) x_{g}$, giving $c_{g} \lambda_{g}\left(c_{g}\right)=1$. We wish to find $b_{g} \in C^{*}$ so that $\sigma\left(b_{g} x_{g}\right)=b_{g} x_{g}$. For this to happen, we must have $c_{g} \lambda_{g}\left(b_{g}\right)=b_{g}$. To produce $b_{g}$ for a fixed $g$, choose $u \in C$ so that if $b_{g}=u+c_{g} \lambda_{g}(u)$, then $b_{g} \neq 0$. Such a $u$ exists; if $c_{g} \neq-1$, then set $u=1$. If $c_{g}=-1$, then let $u \in Z$ be any element with $g(u) \neq u$. This will guarantee that $b_{g}=u+c_{g} \lambda_{g}(u) \neq 0$. We then get

$$
\begin{aligned}
\lambda_{g}\left(b_{g}\right) & =\lambda_{g}(u)+\lambda_{g}^{2}(u) \lambda_{g}\left(c_{g}\right)=\lambda_{g}(u)+c_{g}^{-1} u c_{g} \lambda_{g}\left(c_{g}\right) \\
& =\lambda_{g}(u)+c_{g}^{-1} u .
\end{aligned}
$$

Thus, $c_{g} \lambda_{g}\left(b_{g}\right)=u+c_{g} \lambda_{g}(u)=b_{g}$, as desired. Therefore, if we define new cocycle $\left(\omega^{\prime}, f^{\prime}\right)$ by

$$
\omega_{g}^{\prime}=\operatorname{Int}\left(b_{g}\right) \circ \omega_{g}
$$




$$
f^{\prime}(g, h)=b_{g} \omega_{g}\left(b_{h}\right) f(g, h) b_{g h}^{-1}
$$

and set $x_{g}^{\prime}=b_{g} x_{g}$, then we have the desired condition $\sigma\left(x_{g}^{\prime}\right)=x_{g}^{\prime}$.

Given an involution $\sigma$ on $(C, G,(\omega, f))=\oplus_{g \in G} C x_{g}$, we next determine necessary and sufficient conditions on a generalized cocycle $(\omega, f)$ for $\sigma$ to satisfy $\sigma\left(x_{g}\right)=x_{g}$ for all $g \in G$.

Lemma 2.3 Let $(C, G,(\omega, f))=\oplus_{g} C x_{g}$ be a generalized crossed product. If $\sigma$ is an involution of the first kind on $C$, then $\sigma$ can be extended to $(C, G,(\omega, f))$ with $\sigma\left(x_{g}\right)=x_{g}$ if and only if for all $g, h \in G$,

$$
\begin{aligned}
\omega_{g h}(\sigma(f(g, h))) & =f(h, g), \\
\left(\omega_{g} \circ \sigma\right)^{2} & =\mathrm{id} .
\end{aligned}
$$

Proof. First, suppose that $\sigma$ satisfies $\sigma\left(x_{g}\right)=x_{g}$. For all $c \in C$, we have $\sigma\left(c x_{g}\right)=$ $x_{g} \sigma(c)=\omega_{g}(\sigma(c)) x_{g}$. So,

$$
\begin{aligned}
c x_{g} & =\sigma\left(\sigma\left(c x_{g}\right)\right)=\sigma\left(\omega_{g}(\sigma(c)) x_{g}\right) \\
& =x_{g} \sigma\left(\omega_{g}(\sigma(c))\right) \\
& =\omega_{g}\left(\sigma\left(\omega_{g}(\sigma(c))\right)\right) x_{g} .
\end{aligned}
$$

Thus, $\left(\omega_{g} \circ \sigma\right)^{2}=$ id on $C$.

Next, we have $x_{g} x_{h}=f(g, h) x_{g h}$, so

$$
\sigma\left(x_{g} x_{h}\right)=x_{h} x_{g}=f(h, g) x_{h g}=f(h, g) x_{g h}
$$

and

$$
\begin{aligned}
\sigma\left(x_{g} x_{h}\right) & =\sigma\left(f(g, h) x_{g h}\right)=x_{g h} \sigma(f(g, h)) \\
& =\omega_{g h}(\sigma(f(g, h))) x_{g h} .
\end{aligned}
$$

Therefore,

$$
f(h, g)=\omega_{g h}(\sigma(f(g, h))) .
$$

These calculations clearly reverse to show that given an involution $\sigma$ on $C$, then $\sigma$ extends to $(C, G,(\omega, f))=\oplus_{g} C x_{g}$ with $\sigma\left(x_{g}\right)=x_{g}$ if and only if the cocycle $(\omega, f)$ satisfies

$$
\begin{aligned}
\omega_{g h}(\sigma(f(g, h))) & =f(h, g), \\
\left(\omega_{g} \circ \sigma\right)^{2} & =\mathrm{id}
\end{aligned}
$$

for all $g, h \in G$. 


\section{Involutions on Inertially Split Division Algebras}

Let $(F, v)$ be a Henselian valued field with $\operatorname{char}(\bar{F}) \neq 2$. If $S$ is an inertially split $F$-central division algebra of exponent 2 , we describe the involutions we will use and how we will write $S$ as a generalized crossed product. By abuse of notation, we will write $v$ for the unique extension of $v$ to any finite dimensional division algebra containing $F$.

Let $Z$ be an inertial lift in $S$ of $Z(\bar{S})$. Then $Z / F$ is Abelian Galois with $\operatorname{Gal}(Z / F) \cong$ $\operatorname{Gal}(Z(\bar{S}) / \bar{F}) \cong \Gamma_{S} / \Gamma_{F}$, and $\operatorname{Gal}(Z / F)$ is an elementary Abelian 2-group; these facts can be found in [?, Lemma 5.1, Cor. 6.10]. By Lemma 2.1, there is an involution $\sigma^{*}$ on $S$ with $\left.\sigma^{*}\right|_{Z}=$ id. Let $C=C_{S}(Z)$. Then $C / Z$ is inertial with $\bar{S}=\bar{C}$ by [?, Lemma 1.8]. The involution $\sigma^{*}$ restricts to an involution of the first kind on $C$ since $\left.\sigma^{*}\right|_{Z}=$ id. We may write $S$ as a generalized cocycle $S=(C, G,(\omega, k))$, where $G=\operatorname{Gal}(Z / F)$. Moreover, by Proposition 2.2 , we may assume that $S=\oplus_{g \in G} C y_{g}$ with $\sigma^{*}\left(y_{g}\right)=y_{g}$ for all $g \in G$. As described in the proof of [?, Thm. 5.6(b)] we may factor our generalized cocycle as $(\omega, k)=(\omega, f c)$ such that $(\omega, f)$ is a generalized cocycle with $f(g, h) \in U_{C}$ for all $g, h \in G$ and $c \in Z^{2}\left(G, F^{*}\right)$ is a symmetric cocycle with values in $F^{*}$. Let $I=(C, G,(\omega, f))$. Then $I$ is similar to an inertial division algebra, although $I$ itself need not be a division algebra. The generalized cocycle $(\omega, f)$ satisfies the hypotheses of Lemma 2.3 because $(\omega, f c)$ satisfies them and $c$ is symmetric with values in $F^{*}$. Therefore, there is an involution $\sigma$ on $I=\oplus_{g \in G} C x_{g}$ with $\left.\sigma\right|_{C}=\left.\sigma^{*}\right|_{C}$ and $\sigma\left(x_{g}\right)=x_{g}$.

Our aim is to describe decomposability of $\left(S, \sigma^{*}\right)$ in terms of decomposability of an associated involution on a residue algebra $\bar{I}$. Since $I$ may not be a division algebra, we must define $\bar{I}$. The pair $(\omega, f)$ is a generalized cocycle with $f(g, h) \in U_{C}$ for each $g, h \in G$. Therefore, there is a well defined function $\bar{f}: G \times G \rightarrow \bar{C}^{*}$ given by $\bar{f}(g, h)=\overline{f(g, h)}$. Also, $\omega_{g} \in \operatorname{Aut}_{F}(C)$ with $\left.\omega_{g}\right|_{Z}=g$, and since $v \circ g=v$, we see that $v \circ \omega_{g}$ is a valuation on $C$ that extends $v$. Thus, by [?, Thm.], $v \circ \omega_{g}=v$. This implies that $\omega_{g}$ sends the valuation ring of $C$ to itself, so there is an induced map $\overline{\omega_{g}}: \bar{C} \rightarrow \bar{C}$, and $\overline{\omega_{g}} \in \operatorname{Aut}_{\bar{F}}(\bar{C})$. If we define $\bar{\omega}: G \rightarrow \operatorname{Aut}_{\bar{F}}(\bar{C})$ by $\bar{\omega}_{g}=\bar{\omega}_{g}$, then it is easy to see that $(\bar{\omega}, \bar{f})$ is a generalized cocycle for $G$ with values in $\bar{C}^{*}$. So, we have an $\bar{F}$-central simple algebra $(\bar{C}, G,(\bar{\omega}, \bar{f}))$, and we set $\bar{I}=(\bar{C}, G,(\bar{\omega}, \bar{f}))$. Alternatively, if $V_{F}$ and $V_{C}$ are the valuation rings of $F$ and $C$, respectively, then $A=\oplus_{g \in G} V_{C} x_{g}$ is the unique up to isomorphism Azumaya $V_{F}$-order in $I$, and $A / J(A)=(\bar{C}, G,(\bar{\omega}, \bar{f})$. Finally, we describe the residue involution $\bar{\sigma}$ on $\bar{I}$. The involution $\left.\sigma\right|_{C}$ on the division algebra $C$ induces an involution $\overline{\left.\sigma\right|_{C}}$ on $\bar{C}$. The generalized cocycle $(\bar{\omega}, \bar{f})$ clearly satisfies the hypotheses of Lemma 2.3 , so there is an involution $\bar{\sigma}$ on $\bar{I}=\oplus_{g \in G} \bar{C} \overline{x_{g}}$ with $\left.\bar{\sigma}\right|_{\bar{C}}=\overline{\left.\sigma\right|_{C}}$ and $\bar{\sigma}\left(\overline{x_{g}}\right)=\overline{x_{g}}$.

To help us go between $S$ and $\bar{I}$, we will use the "leading monomial" map on $S$. Recall that on $S=\oplus_{g} C y_{g}$, where $\left.\operatorname{Int}\left(y_{g}\right)\right|_{C}=\omega_{g}$ and $y_{g} y_{h}=(f c)(g, h) y_{g h}$, we have $\left.\operatorname{Int}\left(y_{g}\right)\right|_{z}=g$. Under the map $\theta_{S}: \Gamma_{S} / \Gamma_{F} \rightarrow \operatorname{Gal}(\bar{Z} / \bar{F})$ of [?, Prop. 1.7], we have $\theta_{S}\left(v\left(y_{g}\right)+\Gamma_{F}\right)=\bar{g}$. Therefore, via the identification $\theta_{S}: \operatorname{Gal}(Z / F) \cong \Gamma_{S} / \Gamma_{F}$, we have $v\left(y_{g}\right)+\Gamma_{F}=g$. Consequently, the values $v\left(y_{g}\right)$ are distinct modulo $\Gamma_{F}=\Gamma_{C}$. So, for an arbitrary $a=\sum_{g} a_{g} y_{g} \in S$, we have $v(a)=v\left(a_{g} y_{g}\right)$ for a uniquely determined "monomial" $a_{g} y_{g}$. We set $\mu(a)=a_{g} y_{g}$, and call 
$\mu(a)$ the leading monomial of $a$. We point out the properties of the leading monomial map in the following lemma.

Lemma 3.1 Let $\mu$ be the leading monomial map on $S$. Then $v(\mu(a))=v(a)$. Also, $\mu\left(\sigma^{*}(a)\right)=\sigma^{*}(\mu(a))$. Finally, $\mu(a)=$ au for some 1-unit $u$.

Proof. The first property comes immediately from the definition of $\mu$. For the second, we note that if $a_{g} \in C$, then $\sigma^{*}\left(a_{g} y_{g}\right)=y_{g} \sigma\left(a_{g}\right)=\omega_{g}\left(\sigma\left(a_{g}\right)\right) y_{g}$, so $\sigma^{*}$ takes monomials to monomials. Moreover, Both $\omega_{g}$ and $\sigma$ are value preserving. These two facts imply that $\mu \circ \sigma^{*}=\sigma^{*} \circ \mu$. Lastly, $\mu(a) a^{-1}$ is a 1-unit because $v(\mu(a))=v(a)$ and $a$ is the sum of $\mu(a)$ and other terms of value strictly greater than $v(\mu(a))$.

The leading monomial map is not multiplicative. However, as a consequence of the lemma above, we have $\mu(a b) \equiv a b \bmod \left(U_{1, S}\right)$.

The involutions we have constructed are all of the first kind. We note in the next result that all them are of the same type.

Proposition 3.2 The involutions $\sigma^{*}, \sigma,\left.\sigma\right|_{C}$, and $\bar{\sigma}$ are all of the same type.

Proof. We prove this by considering the following situation: $T=(C, G,(\omega, f))=\oplus_{g} C x_{g}$ and $\sigma$ is an involution on $T$ that is trivial on $Z$ with $\sigma\left(x_{g}\right)=x_{g}$. Then $\sigma\left(c_{g} x_{g}\right)=x_{g} \sigma\left(c_{g}\right)=$ $\omega_{g}\left(\sigma\left(c_{g}\right)\right) x_{g}$. Let $\lambda_{g}=\omega_{g} \circ \sigma$. Then, by Lemma 2.3, we see that $\lambda_{g}^{2}=$ id. The map $\lambda_{g}$ is an antiautomorphism of $C$, so $\lambda_{g}$ is an involution of $C$. Note that $\left.\lambda_{g}\right|_{Z}=g$, so $\lambda_{g}$ is an involution of the second kind when $g \neq 1$. Also, $\sigma\left(c_{g} x_{g}\right)=c_{g} x_{g}$ if and only if $\lambda_{g}\left(c_{g}\right)=c_{g}$. Consequently, $\operatorname{Sym}(T, \sigma)=\oplus_{g} \operatorname{Sym}\left(C, \lambda_{g}\right) x_{g}$. Let $n=\operatorname{deg}(T)$ and $m=\operatorname{deg}(C)$. Then

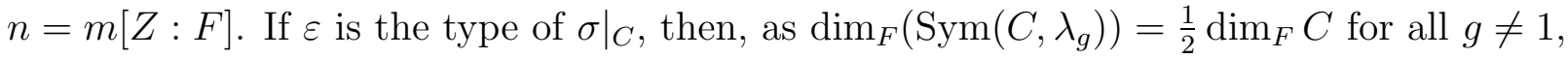

$$
\begin{aligned}
\operatorname{dim}_{F}(\operatorname{Sym}(T, \sigma)) & =\sum_{g \in G} \operatorname{dim}_{F}\left(\operatorname{Sym}\left(C, \lambda_{g}\right)\right) \\
& =\operatorname{dim}_{F}\left(\operatorname{Sym}\left(C,\left.\sigma\right|_{C}\right)+(|G|-1) \frac{1}{2} \operatorname{dim}_{F}(C)\right. \\
& =[Z: F]\left(\frac{1}{2} m(m+\varepsilon)+([Z: F]-1) \frac{1}{2} m^{2}\right) \\
& =\frac{1}{2} n(m+\varepsilon)+\frac{1}{2} n^{2}-\frac{1}{2} n m=\frac{1}{2} n(n+\varepsilon) .
\end{aligned}
$$

Therefore, the type of $\sigma$ on $T$ is $\varepsilon$. We can apply this result to $S$ and to $I$ to get that the types of $\sigma$ and $\sigma^{*}$ are equal to the type of $\left.\sigma\right|_{C}$. Also, applying it to $\bar{I}=(\bar{C}, G,(\bar{\omega}, \bar{f}))$, we see that the type of $\bar{\sigma}$ is equal to the type of $\left.\bar{\sigma}\right|_{C}$, which is equal to the type of $\left.\sigma\right|_{C}$, by [?, $\S 1$, Prop. 3]. This shows that the types of $\sigma^{*}, \sigma,\left.\sigma\right|_{C}$, and $\bar{\sigma}$ are all equal.

We now relate the discriminants of the various involutions. Recall that if $(T, \tau)$ is a central simple algebra with involution of the first kind, then the discriminant $\operatorname{disc}(\tau)$ of $\tau$ is defined as follows. If $\varepsilon= \pm 1$ is the type of $\tau$, then $\operatorname{disc}(\tau)=(-1)^{\operatorname{deg}(T)} \operatorname{Nrd}(a) F^{* 2} \in F^{*} / F^{* 2}$ for any $a \in T^{*}$ with $\tau(a)=-\varepsilon a$. This definition, for $\varepsilon=1$, can be found in [?, Def. 7.2], 
and for general $\varepsilon$ in [?, p. 94]. To define a notation used in the following proposition, recall that there is a split exact sequence

$$
1 \longrightarrow U_{F} / U_{F}^{2} \longrightarrow F^{*} / F^{* 2} \longrightarrow \Gamma_{F} / 2 \Gamma_{F} \longrightarrow 1,
$$

and $U_{F} / U_{F}^{2} \cong \bar{F}^{*} / \bar{F}^{* 2}$ since $F$ is Henselian with $\operatorname{char}(\bar{F}) \neq 2$. Therefore, there is a group monomorphism $i: \bar{F}^{*} / \bar{F}^{* 2} \rightarrow F^{*} / F^{* 2}$ given by $i\left(\bar{u} \bar{F}^{* 2}\right)=u F^{* 2}$ for any lift $u \in U_{F}$ of $\bar{u}$.

Proposition 3.3 We have $\operatorname{disc}\left(\sigma^{*}\right)=\operatorname{disc}(\sigma)=N_{Z / F}\left(\operatorname{disc}\left(\left.\sigma\right|_{C}\right)\right)$, and these are equal to $i(\operatorname{disc}(\bar{\sigma}))$.

Proof. Let $n=\operatorname{deg}(S)$ and $m=\operatorname{deg}(C)$, and let $\varepsilon$ be the type of $\sigma$. If $a \in C^{*}$ with $\sigma(a)=-\varepsilon a$, then $\operatorname{disc}\left(\left.\sigma\right|_{C}\right)$ is $(-1)^{m} \operatorname{Nrd}_{C}(a)$. Since we know, by Proposition 3.2, that the types of $\sigma^{*}, \sigma$, and $\left.\sigma\right|_{C}$ are all the same, we have $\operatorname{disc}\left(\sigma^{*}\right)=(-1)^{n} \operatorname{Nrd}_{S}(a)$ and $\operatorname{disc}(\sigma)=(-1)^{n} \operatorname{Nrd}_{I}(a)$. However,

$$
\operatorname{Nrd}_{A}(a)=N_{Z / F}\left(\operatorname{Nrd}_{C}(a)\right)=\operatorname{Nrd}_{I}(a)
$$

so $\operatorname{disc}\left(\sigma^{*}\right)=N_{Z / F}\left(\operatorname{disc}\left(\left.\sigma\right|_{C}\right)\right)=\operatorname{disc}(\sigma)$. The equality $\operatorname{disc} \sigma=i(\operatorname{disc} \bar{\sigma})$ is proved in [?, $\S 2$, Prop. 3].

\section{Decompositions of Involutions}

In this section we prove the main theorems of this paper. We continue to use the same notation as in the previous section: $F$ is a Henselian valued field with $\operatorname{char}(\bar{F}) \neq 2, S=$ $(C, G,(\omega, f c))$ is an inertially split division algebra, $I=(C, G,(\omega, f))$ is similar to an inertial division algebra, and $\bar{I}=(\bar{C}, G,(\bar{\omega}, \bar{f}))$. We have involutions $\sigma^{*}$ on $S$ and $\sigma$ on $I$ with $\left.\sigma^{*}\right|_{C}=$ $\left.\sigma\right|_{C}$ an involution of the first kind on $C$. Moreover, if $S=\oplus_{g \in G} C y_{g}$ with $\left.\operatorname{Int}\left(y_{g}\right)\right|_{C}=\omega_{g}$ and $y_{g} y_{h}=(f c)(g, h) y_{g h}$, and if $I=\oplus_{g \in G} C x_{g}$ with $\left.\operatorname{Int}\left(x_{g}\right)\right|_{C}=\omega_{g}$ and $x_{g} x_{h}=f(g, h) x_{g h}$, then $\sigma^{*}\left(a y_{g}\right)=\omega_{g}(\sigma(a)) y_{g}$ and $\sigma\left(a y_{g}\right)=\omega_{g}(\sigma(a)) x_{g}$ for all $a \in C$. We have an induced involution $\bar{\sigma}$ on $\bar{I}=(\bar{C}, G,(\bar{\omega}, \bar{f}))$ that satisfies $\bar{\sigma}\left(\overline{a x_{g}}\right)=\bar{\omega}_{g}(\bar{\sigma}(\bar{a})) \overline{x_{g}}$.

Let $K=F\left(\sqrt{a_{1}}, \ldots, \sqrt{a_{n}}\right)$ be an elementary Abelian extension of $F$ of degree $2^{n}$ and let $A$ be an $F$-central simple algebra of degree $2^{n}$ containing $K$. If $A$ decomposes into a tensor product of quaternion algebras as $A=\left(a_{1}, b_{1}\right) \otimes_{F} \cdots \otimes_{F}\left(a_{n}, b_{n}\right)$ for some $b_{i} \in F^{*}$, we say that $A$ has a decomposition adapted to $K$ into quaternion algebras.

We now extend [?, Thm. 4.1] to the case of inertially split division algebras over a Henselian valued field of residue characteristic not 2. To help with the argument, we point out that if $Q$ is a quaternion algebra with an involution $\sigma$ of the first kind, then $Q$ has quaternion generators $i$ and $j$ with $\sigma(i)= \pm i$ and $\sigma(j)= \pm j$. We give a proof of this fact for the convenience of the reader. If $\sigma$ is symplectic, then this is clear. If $\sigma$ is orthogonal, let $\gamma$ be the unique symplectic involution on $Q$. There is an element $v$ with $\gamma(v)=-v$ and $\sigma=\operatorname{Int}(v) \circ \gamma$. Recall that the square of any element in $\operatorname{Skew}(Q, \gamma)$ is in $F$. By dimension 
count, there is a $\sigma$-symmetric element $u \in \operatorname{Skew}(Q, \gamma)$. Then $u^{2} \in F$ and

$$
u=\sigma(u)=v \gamma(u) v^{-1}=-v u v^{-1},
$$

so $u$ and $v$ anticommute. Then $u$ and $v$ are quaternion generators of $Q$ with $\sigma(u)=u$ and $\sigma(v)=-v$.

Theorem 4.1 The following statements are equivalent.

1. $S$ contains an F-central quaternion algebra stable under $\sigma^{*}$;

2. $\bar{I}$ contains an $\bar{F}$-central quaternion algebra generated by monomials $\overline{a x_{g}}$ and $\bar{b} \overline{x_{h}}$ that are each either symmetric or skew-symmetric with respect to $\bar{\sigma}$;

3. $\bar{I}$ contains an $\bar{F}$-central quaternion algebra $Q$ stable under $\bar{\sigma}$ with $[Q \cap \bar{Z}: \bar{F}]=2$ and $\left[C_{\bar{I}}(Q) \cap \bar{Z}: \bar{F}\right]=\frac{1}{2}[\bar{Z}: \bar{F}] ;$

4. $\bar{Z}$ contains a quadratic extension $L$ of $\bar{F}$ such that $C_{\bar{I}}(L)=L \otimes_{\bar{F}} A$ for some $\bar{F}$-central subalgebra $A$ of $\bar{I}$ that is stable under $\bar{\sigma}$ and with $[A \cap \bar{Z}: \bar{F}]=\frac{1}{2}[\bar{Z}: \bar{F}]$.

Proof. $(1) \Rightarrow(2)$ : Let $Q$ be an $F$-central quaternion subalgebra of $S$ stable under $\sigma^{*}$. As pointed out above, we may assume that $Q$ has generators $i$ and $j$ with $\sigma^{*}(i)= \pm i$ and $\sigma^{*}(j)= \pm j$. We may write $i=a y_{g} v_{g}$ and $j=b y_{h} v_{h}$ with $a, b \in C$ and $v_{g}, v_{h}$ 1-units in $S$ by Lemma 3.1. We may also assume that $a$ and $b$ are units in $C$ since $\Gamma_{C}=\Gamma_{F}$ and scalar multiples of quaternion generators are still quaternion generators. For convenience we view $\omega_{g}$ as acting on $S$ via $\operatorname{Int}\left(y_{g}\right)$. Since $i^{2} \in F^{*}$, we have

$$
\begin{aligned}
i^{2} & =\left(a y_{g} v_{g}\right)\left(a y_{g} v_{g}\right)=a \omega_{g}\left(v_{g} a\right)(f c)(g, g) v_{g} \\
& =a \omega_{g}\left(v_{g}\right) \omega_{g}(a) f(g, g) c(g, g) v_{g} .
\end{aligned}
$$

Therefore, as $c(g, g) \in F^{*}$, we have $a \omega_{g}\left(v_{g}\right) \omega_{g}(a) f(g, g) v_{g} \in F$. Moreover, this element is a unit in $S$. Therefore, taking residues and using the fact that $v_{g}$ is a 1-unit, we have $\overline{a \omega_{g}}(\bar{a}) \bar{f}(g, g) \in \bar{F}^{*}$. This shows that $\overline{a x_{g}} \in \bar{I}$ satisfies $\left(\overline{a x_{g}}\right)^{2} \in \bar{F}^{*}$. Similarly, $\left(\bar{b} \overline{x_{h}}\right)^{2} \in \bar{F}^{*}$. Furthermore, we have $j i=-i j$, and simplifying the equation

$$
\left(b y_{h} v_{h}\right)\left(a y_{g} v_{g}\right)=-\left(a y_{g} v_{g}\right)\left(b y_{h} v_{h}\right)
$$

gives

$$
b \omega_{h}\left(v_{h} a\right)(f c)(h, g) y_{h g} v_{g}=-a \omega_{g}\left(v_{g} b\right)(f c)(g, h) y_{g h} v_{h} .
$$

Since $g h=h g$ and $c(g, h)=c(h, g) \in F^{*}$, this yields

$$
b \omega_{h}\left(v_{h} a\right) f(h, g) \omega_{g h}\left(v_{g}\right)=-a \omega_{g}\left(v_{g} b\right) f(g, h) \omega_{g h}\left(v_{h}\right) .
$$


Again, both sides are units, and by taking residues we get $\bar{b} \overline{\omega_{h}}(\bar{a}) \bar{f}(h, g)=-\overline{a \omega_{g}}(\bar{b}) \bar{f}(g, h)$. Therefore, $\overline{a x_{g}}$ and $\bar{b} \overline{x_{h}}$ anticommute. They then generate an $\bar{F}$-central quaternion algebra in $\bar{I}$. For stability under $\bar{\sigma}$ of this quaternion algebra, since $\sigma^{*}(i)= \pm i$, we have

$$
\pm a y_{g} v_{g}=\sigma^{*}\left(a y_{g} v_{g}\right)=\sigma^{*}\left(v_{g}\right) \omega_{g}(\sigma(a)) y_{g}
$$

or $\sigma^{*}\left(v_{g}\right) \omega_{g}(\sigma(a))= \pm a \omega_{g}\left(v_{g}\right)$. Taking residues, we get $\overline{\omega_{g}}(\bar{\sigma}(\bar{a}))= \pm \bar{a}$ in $\bar{S}=\bar{C}$. Therefore, $\bar{\sigma}\left(\overline{a x_{g}}\right)= \pm \overline{a x_{g}}$. Similarly, $\bar{\sigma}\left(\bar{b} \overline{x_{h}}\right)= \pm \bar{b} \overline{x_{h}}$. Therefore, $\bar{I}$ contains an $\bar{F}$-central quaternion algebra stable under $\bar{\sigma}$ that is generated by monomials, both of which are either symmetric or skew under $\bar{\sigma}$.

$(2) \Rightarrow(1)$ : Let $\widetilde{Q}$ be an $\bar{F}$-central quaternion algebra in $\bar{I}$ with quaternion generators $\overline{a x_{g}}$ and $\bar{b} \overline{x_{h}}$ satisfying $\bar{\sigma}\left(\overline{a x_{g}}\right)= \pm \overline{a x_{g}}$ and $\bar{\sigma}\left(\bar{b} \overline{x_{h}}\right)= \pm \bar{b} \overline{x_{h}}$. Let $z$ be a unit in $Z$ such that $g(z)=-z$ and $g^{\prime}(z)=z$ for all $g^{\prime} \neq g$ in $G$. Then $z^{2} \in F^{*}$; moreover, $g(\bar{z})=-\bar{z}$ and $\bar{z}^{2} \in \bar{F}^{*}$. Thus, $\left(\overline{a x_{g}}\right) \bar{z}=-\bar{z}\left(\overline{a x_{g}}\right)$. Therefore, $\overline{a x_{g}}$ and $\bar{z}$ generate an $\bar{F}$-central quaternion algebra in $\bar{I}$ that satisfies the same conditions as $\widetilde{Q}$. If we can find a lift $a^{\prime} \in C$ of $\bar{a}$ with $\left(a^{\prime} y_{g}\right)^{2} \in F$ and $\sigma^{*}\left(a^{\prime} y_{g}\right)= \pm a^{\prime} y_{g}$, then $a^{\prime} x_{g}$ and $z$ will generate a $\sigma^{*}$-stable quaternion algebra in $S$.

We first find a lift $a^{\prime}$ of $\bar{a}$ that satisfies $\left(a^{\prime} y_{g}\right)^{2} \in F$. Set $H=\langle g\rangle$, and let $a$ be any lift of $\bar{a}$ in $C$. We have $\left(a y_{g}\right)^{2}=a \omega_{g}(a) f(g, g) c(g, g)$. If $\left(\overline{a x_{g}}\right)^{2}=\bar{\alpha}$, then $\overline{a \omega_{g}}(\bar{a}) \bar{f}(g, g)=\bar{\alpha}$. Therefore, $a \omega_{g}(a) f(g, g)=\alpha u$ for some 1-unit $u$ in $C$. We consider the restricted generalized cocycle $\left(\omega^{\prime}, f^{\prime}\right)=\operatorname{res}_{H}^{G}(\omega, f) \in \mathcal{Z}\left(H, C^{*}\right)$. By setting $a_{g}=a$ and $a_{\text {id }}=1$, we get a cocycle $(\theta, e)$ equivalent to $\left(\omega^{\prime}, f^{\prime}\right)$, defined by

$$
\begin{aligned}
\theta_{h} & =\operatorname{Int}\left(a_{h}\right) \circ \omega_{h}^{\prime}, \\
e(h, k) & =a_{h} \omega_{h}^{\prime}\left(a_{k}\right) f^{\prime}(h, k) a_{h k}^{-1}
\end{aligned}
$$

for all $h, k \in H$. If $\alpha^{\prime}$ is the normalized cocycle in $Z^{2}\left(H, F^{*}\right)$ with $\alpha^{\prime}(g, g)=\alpha$, then $\left(\theta, e / \alpha^{\prime}\right)$ is a generalized cocycle. If we set $u^{\prime}=e / \alpha^{\prime}$, then $u^{\prime}(g, g)=u$. We thus have $H=\operatorname{Gal}\left(Z / Z^{g}\right)$ and a generalized cocycle $(\theta, u) \in \mathcal{Z}\left(H, C^{*}\right)$ with values in the group of 1-units of $C$. By [?, Thm. 1.1], there is a group homomorphism $\psi: H \rightarrow \operatorname{Aut}(C)$ with $\left(\theta, u^{\prime}\right) \sim(\psi, 1)$. Therefore, there is a $b_{g} \in U_{1, C}$ with $b_{g} \theta_{g}\left(b_{g}\right) u^{\prime}(g, g)=1$. Replacing $a$ by $a^{\prime}=b_{g} a$ then gives a monomial $a^{\prime} y_{g}$ that satisfies $\left(a^{\prime} y_{g}\right)^{2}=\alpha c(g, g) \in F^{*}$. Note that $\overline{a^{\prime}}=\bar{a}$ since $b$ is a 1-unit.

We now adjust our monomial $M=a^{\prime} y_{g}$ to get a monomial $N$ with $\sigma^{*}(N)= \pm N$, but preserving the property $N^{2} \in F^{*}$. Set $\beta=\alpha c(g, g)$. Recall that since $\bar{\sigma}\left(\overline{a x_{g}}\right)=\varepsilon \overline{a x_{g}}$, where $\varepsilon= \pm 1$, we have $\overline{\omega_{g}}(\bar{\sigma}(\bar{a}))=\varepsilon \bar{a}$. Moreover,

$$
\sigma^{*}(M)=\omega_{g}\left(\sigma\left(a^{\prime}\right)\right) y_{g}=\left(\omega_{g}\left(\sigma\left(a^{\prime}\right)\right)\left(a^{\prime}\right)^{-1}\right) a^{\prime} y_{g}
$$

Set $v=\varepsilon \omega_{g}\left(\sigma\left(a^{\prime}\right)\right)\left(a^{\prime}\right)^{-1}$. Then $v$ is a 1-unit in $C$, and $\sigma^{*}(M)=\varepsilon v M$. Let $\psi=\left.\operatorname{Int}(M)\right|_{F(v)}$. Since $M^{2}=\beta \in F$, we have $\sigma^{*}(M)^{2}=\beta$, which yields $v \psi(v)=1$. Note that $\sigma^{*}(v)=v$ is an easy consequence of the equations $\sigma^{*}(M)=\varepsilon v M$ and $v \psi(v)=1$. We will produce an 
$s \in F(v)$ with $\sigma^{*}(s M)= \pm s M$ and $(s M)^{2} \in F^{*}$. Because $\left.\sigma^{*}\right|_{F(v)}=\mathrm{id}$, these conditions are equivalent to

$$
\begin{aligned}
& v \psi(s)= \pm s, \\
& s \psi(s) \in F^{*} .
\end{aligned}
$$

The equation $v \psi(v)=1$ gives $\psi(v)=v^{-1}$, which implies that $\psi$ is an $F$-automorphism of the field $F(v) \subseteq C$. Moreover, $\psi^{2}=$ id since $M^{2} \in F^{*}$. We have a 1-cocycle $\varphi \in H^{1}\left(\langle\psi\rangle, U_{1, F(v)}\right)$ given by $\varphi(1)=1$ and $\varphi(g)=v$. Since $U_{1, F(v)}$ is uniquely divisible by $2=|\langle\psi\rangle|$, this cohomology group is trivial. Thus, there is a $w \in U_{1, F(v)}$ with $v=w / \psi(w)$. Since $w$ is a 1-unit, $w \psi(w)$ is also a 1-unit, so there is a $t \in U_{1, F(v)}$ with $w \psi(w)=t$. If $s=w / t$, then a short calculation shows that $v \psi(s)= \pm s$ and $s \psi(s)= \pm 1$. Therefore, the monomial $s M$ satisfies $(s M)^{2}= \pm \beta$ and $\sigma^{*}(s M)= \pm s M$. Thus, as mentioned in the first paragraph of $(2) \Rightarrow(1), s M=s a^{\prime} y_{g}$ and $z$ are monomials that generate a $\sigma^{*}$-invariant $F$-central quaternion algebra in $S$.

$(2) \Rightarrow(3)$ : Let $\widetilde{Q}$ be an $\bar{F}$-central quaternion algebra in $\bar{I}$ with quaternion generators $\overline{a x_{g}}$ and $\bar{b} \overline{x_{h}}$ that are each either symmetric or skew with respect to $\bar{\sigma}$. Let $z$ be a unit in $Z$ such that $g(z)=-z$ and $g^{\prime}(z)=z$ for all $g^{\prime} \neq g$. Then $z^{2} \in F$. Moreover, $\left(\overline{a x_{g}}\right) \bar{z}=-\bar{z}\left(\overline{a x_{g}}\right)$. So, $\overline{a x_{g}}$ and $\bar{z}$ generate an $\bar{F}$-central quaternion algebra $Q^{\prime}$ in $\bar{I}$ with the same properties as $\widetilde{Q}$. We have $Q^{\prime} \cap \bar{Z}=\bar{F}(\bar{z})$, so $\left[Q^{\prime} \cap \bar{Z}: \bar{F}\right]=2$. Also, it is clear that $C_{\bar{I}}\left(Q^{\prime}\right) \cap \bar{Z}=\bar{Z}^{g}$ since $\operatorname{Int}\left(\overline{a x_{g}}\right)=\operatorname{Int}\left(\overline{x_{g}}\right)$ induces $g$ on $\bar{Z}$. So, $\left[C_{\bar{I}}\left(Q^{\prime}\right) \cap \bar{Z}: \bar{F}\right]=\frac{1}{2}[\bar{Z}: \bar{F}]$.

$(3) \Rightarrow(2)$ : Let $Q$ be a $\bar{\sigma}$-stable $\bar{F}$-central quaternion algebra in $\bar{I}$ that contains a quadratic extension $L$ of $\bar{F}$ inside $\bar{Z}$ and for which $\left[C_{\bar{I}}(Q) \cap \bar{Z}: \bar{F}\right]=\frac{1}{2}[\bar{Z}: \bar{F}]$. Set $L^{\prime}=C_{\bar{I}}(Q) \cap \bar{Z}$. Then, by dimension count, $\bar{Z}=L \otimes_{\bar{F}} L^{\prime}$. Let $g \in \operatorname{Gal}(\bar{Z} / \bar{F})$ satisfy $L^{\prime}=\bar{Z}^{g}$ and $\left.g\right|_{L} \neq$ id. Choose $\bar{z} \in L$ with $g(\bar{z})=-\bar{z}$. Then there is a $j \in Q$ with $j \bar{z}=-\bar{z} j$. Moreover, $\operatorname{Int}(j)$ is the identity on $L^{\prime}$ since $L^{\prime} \subseteq C_{\bar{I}}(Q)$. So, $\operatorname{Int}(j)$ is equal to $g$ on $\bar{Z}$. Thus, $\operatorname{Int}\left(\overline{x_{g}} j^{-1}\right)$ is the identity on $\bar{Z}$, so $\overline{x_{g}} j^{-1} \in C_{\bar{I}}(\bar{Z})=\bar{C}$, and so $\overline{x_{g}} j^{-1}=\bar{u}$ for some $u \in C$. Thus, $j=\overline{u x_{g}}$ is a monomial. We now show that we can alter $j$ to assume $\bar{\sigma}(j)= \pm j$. Since $Q$ is stable under $\bar{\sigma}$ and $\bar{\sigma}$ is trivial on $\bar{Z}$, we see that $\bar{\sigma}(j) \bar{z} \bar{\sigma}(j)^{-1}=j \bar{z} j^{-1}$. Thus, $\bar{\sigma}(j)=b j$ for some $b \in L=\bar{F}(\bar{z})$. Then $j=\bar{\sigma}(b j)=b j b=b g(b) j$. Therefore, $b g(b)=1$, so $b=b^{\prime} / g\left(b^{\prime}\right)$ for some $b^{\prime} \in L$ by Hilbert Theorem 90. Then $b^{\prime} j$ is a new monomial that satisfies $\bar{\sigma}\left(b^{\prime} j\right)=b^{\prime} j$ and $\left(b^{\prime} j\right)^{2}=j^{2} \in \bar{F}$. The monomials $\bar{z}$ and $b^{\prime} j$ then generate a $\bar{\sigma}$-stable quaternion subalgebra of $\bar{I}$.

$(3) \Leftrightarrow(4)$ : Suppose that $Q$ is a $\bar{\sigma}$-stable $\bar{F}$-central quaternion subalgebra of $\bar{I}$ with $[Q \cap \bar{Z}: \bar{F}]=2$ and $\left[C_{\bar{I}}(Q) \cap \bar{Z}: \bar{F}\right]=\frac{1}{2}[\bar{Z}: \bar{F}]$. If we set $L=Q \cap \bar{Z}$, then $C_{\bar{I}}(L)=$ $L \otimes_{\bar{F}} C_{\bar{I}}(Q) \subseteq Q \otimes_{\bar{F}} C_{\bar{I}}(Q)$. Moreover, since $Q$ is stable under $\bar{\sigma}$, its centralizer $C_{\bar{I}}(Q)$ is also stable under $\bar{\sigma}$. Therefore, setting $A=C_{\bar{I}}(Q)$ gives (4). Conversely, if $L \subseteq \bar{Z}$ is a quadratic extension of $\bar{F}$ with $C_{\bar{I}}(L)=L \otimes_{\bar{F}} A$ for some $\bar{\sigma}$-stable $\bar{F}$-central subalgebra $A$ that satisfies $[A \cap \bar{Z}: \bar{F}]=\frac{1}{2}[\bar{Z}: \bar{F}]$, then we may write $\bar{I}=C_{\bar{I}}(A) \otimes_{\bar{F}} A$ by the double centralizer theorem, and $L \subseteq C_{\bar{I}}(A)$. Furthermore, dimension count shows that $C_{\bar{I}}(A)$ is a quaternion algebra. It is stable under $\bar{\sigma}$ since $A$ is stable under $\bar{\sigma}$. Finally, $C_{\bar{I}}(Q)=A$ by the double centralizer 
theorem, so $\left[C_{\bar{I}}(Q) \cap \bar{Z}: \bar{F}\right]=[A \cap \bar{Z}: \bar{F}]=\frac{1}{2}[\bar{Z}: \bar{F}]$.

The next two results will be used in the proof of Proposition 4.4 below. That proposition is a special case of the second of our main results, Theorem 4.5, and it will be used in the proof of Theorem 4.5.

Lemma 4.2 Let $J$ be an inertial F-central division algebra. If $\tau$ is an involution on $\bar{J}$ of the first kind, then there is a unique up to isomorphism involution $\sigma$ on $J$ with $\bar{\sigma}=\tau$.

Proof. By [?, $\S 1$, Prop. 4], there is an involution $\sigma$ on $J$ with $\bar{\sigma}=\tau$. Suppose that $\sigma^{\prime}$ is a second lift of $\tau$. Then $\bar{\sigma}=\overline{\sigma^{\prime}}=\tau$. There is a $u \in J$ with $\sigma(u)= \pm u$ and $\sigma^{\prime}=\operatorname{Int}(u) \circ \sigma$. Since $\Gamma_{J}=\Gamma_{F}$, we may assume that $u$ is a unit. Then $\tau=\operatorname{Int}(\bar{u}) \circ \tau$, so $\bar{u} \in \bar{F}^{*}$. We then may further modify $u$ to assume that $\bar{u}=\overline{1}$. By Hensel's lemma, $u=x^{2}$ for some $x \in F(u) \subseteq J$. Moreover, since the type of $\sigma$ and $\sigma^{\prime}$ are the same, being equal to the type of $\tau$ by [?, $\S 1$, Prop. 3], $\sigma(u)=u$. This yields $\sigma(x)=x$, so $u=\sigma(x) x$. Therefore, $\sigma^{\prime}=\operatorname{Int}(\sigma(x) x) \circ \sigma$, which proves that $\sigma^{\prime}$ and $\sigma$ are isomorphic as involutions.

Proposition 4.3 Let $J$ be an F-central inertial division algebra and let $\sigma$ be an involution of the first kind on $J$. If $\widetilde{B}$ is an $\bar{F}$-central subalgebra of $\bar{J}$ with $\bar{\sigma}(\widetilde{B})=\widetilde{B}$, then there is an inertial lift $B$ of $\widetilde{B}$ in $J$ with $\sigma(B)=B$.

Proof. Let $B$ be an inertial lift of $\widetilde{B}$ in $J$; existence of $B$ follows from [?, Thm. 2.9]. Set $B^{\prime}=C_{J}(B)$. Then $J=B \otimes_{F} B^{\prime}$ by the double centralizer theorem. Also, $\overline{B^{\prime}}=C_{\bar{J}}(\widetilde{B})$ and $\bar{J}=\bar{B} \otimes_{\bar{F}} \overline{B^{\prime}}$. By hypothesis, $\bar{\sigma}$ restricts to an involution on $\bar{B}$, and so it also restricts to an involution on $\overline{B^{\prime}}=C_{\bar{J}}(\bar{B})$. We have $\exp (B)=\exp (\bar{B}) \leq 2$ by the isomorphism $\operatorname{IBr}(F) \rightarrow \operatorname{Br}(\bar{F})$ of [?, Thm. 2.8], so $B$ has an involution of the first kind. Similarly, $B^{\prime}$ has an involution of the first kind. Then, by [?, §1, Prop. 4], there are involutions $\tau$ and $\rho$ on $B$ and $B^{\prime}$, respectively, with $\bar{\tau}=\left.\bar{\sigma}\right|_{\bar{B}}$ and $\bar{\rho}=\left.\bar{\sigma}\right|_{\overline{B^{\prime}}}$. By [?, $\S 1$, Prop. 3], $\tau$ and $\bar{\tau}$ are of the same type, $\sigma$ and $\bar{\sigma}$ are of the same type, and $\rho$ and $\bar{\rho}$ are of the same type. Therefore, as $\bar{\sigma}=\left.\left.\bar{\sigma}\right|_{\bar{B}} \otimes \bar{\sigma}\right|_{\bar{B}^{\prime}}$, the involutions $\sigma$ and $\tau \otimes \rho$ are of the same type on $J$. Thus, there is a $u \in J^{*}$ with $\sigma(u)=u$ and $\tau \otimes \rho=\operatorname{Int}(u) \circ \sigma$. We may assume that $v(u)=0$ since $\Gamma_{J}=\Gamma_{F}$. So, $\overline{\tau \otimes \rho}=\operatorname{Int}(\bar{u}) \circ \bar{\sigma}$. However, since $\tau$ and $\rho$ both reduce to $\bar{\sigma}$, this gives $\bar{\sigma}=\operatorname{Int}(\bar{u}) \circ \bar{\sigma}$. From this we see that $\bar{u} \in \bar{F}^{*}$. So, we may further modify $u$ to assume that $\bar{u}=\overline{1}$. Since $\operatorname{char}(\bar{F}) \neq 2$, there is an $x \in F(u) \subseteq J$ with $u=x^{2}$. This forces $\sigma(x)=x$. In particular, we see that $\operatorname{Int}(u)=\operatorname{Int}(\sigma(x) x))$. This yields

$$
B=\rho(B)=u \sigma(B) u^{-1}=(\sigma(x) x) B(\sigma(x) x)^{-1},
$$

which then implies that $\sigma(B)=(\sigma(x) x)^{-1} B \sigma(x) x$, so $\sigma\left(x^{-1} B x\right)=x^{-1} B x$. Therefore, $x^{-1} B x$ is an inertial lift of $\widetilde{B}$ that is stable under $\sigma$.

As mentioned earlier, the following proposition will be used in the proof of Theorem 4.5.

Proposition 4.4 Let $J$ be an F-central inertial division algebra and let $\sigma$ be an involution of the first kind on $J$. Then $(J, \sigma)$ decomposes into a tensor product of quaternion algebras 
if and only if $(\bar{J}, \bar{\sigma})$ decomposes into a tensor product of quaternion algebras. Furthermore, if $J$ contains a maximal subfield $K$ that is an elementary Abelian 2-extension of $F$, then $J$ has a decomposition adapted to $K$ into quaternion algebras stable under $\sigma$ if and only if $\bar{J}$ has a decomposition adapted to $\bar{K}$ into quaternion algebras stable under $\bar{\sigma}$.

Proof. First note that the second statement implies the first since any tensor product of quaternion algebras has a decomposition adapted to an appropriate Kummer extension. More concretely, if $A=\left(a_{1}, b_{1}\right) \otimes_{F} \cdots \otimes_{F}\left(a_{n}, b_{n}\right)$ is a division algebra, then $A$ has a decomposition adapted to $F\left(\sqrt{a_{1}}, \ldots, \sqrt{a_{n}}\right)$. We therefore prove only the second statement.

Let $K$ be an elementary Abelian 2-extension of $F$ that is a maximal subfield of $J$. Note that $K / F$ is inertial since $J / F$ is inertial. If $J=\otimes_{i=1}^{n} Q_{i}$ is a decomposition adapted to $K$ into $\sigma$-stable quaternion algebras, then there are quadratic subextensions $K_{i}$ of $K$ with $K=K_{1} \cdots K_{n}$ and $K_{i} \subseteq Q_{i}$. Since $J / F$ is inertial, it is clear that $\bar{J}=\otimes_{i=1}^{n} \overline{Q_{i}}$, and $\bar{\sigma}\left(\overline{Q_{i}}\right)=\overline{Q_{i}}$ since $\sigma\left(Q_{i}\right)=Q_{i}$. Moreover, $\overline{K_{i}} \subseteq \overline{Q_{i}}$, so this decomposition of $\bar{J}$ is adapted to $\bar{K}$.

Conversely, suppose that $\bar{J}=\otimes_{i=1}^{n} \widetilde{Q}_{i}$ is a tensor product of $\bar{\sigma}$-stable quaternion algebras, and that this decomposition is adapted to $\bar{K}$. There are quadratic subextensions $\widetilde{K}_{i}$ of $\bar{K}$ with $\widetilde{K}_{i} \subseteq \widetilde{Q_{i}}$. By Proposition 4.3, there are $\sigma$-stable quaternion algebras $Q_{i} \subseteq J$ with $\overline{Q_{i}}=\widetilde{Q_{i}}$. Let $\sigma_{i}=\left.\sigma\right|_{Q_{i}}$. If $J^{\prime}=\otimes_{i=1}^{n} Q_{i}$ and $\sigma^{\prime}=\otimes_{i=1}^{n} \sigma_{i}$, then $J^{\prime}$ is a division algebra with $\overline{J^{\prime}}=\bar{J}$ by [?, Thm. 1]. By the uniqueness of inertial lifts [?, Thm. 2.8], we have $J^{\prime} \cong J$. We then identify $J^{\prime}=J$. From this we see that $\overline{\sigma^{\prime}}=\bar{\sigma}$, so, by Lemma $4.2, \sigma^{\prime}$ and $\sigma$ are isomorphic involutions on $J$. Since $\left(J, \sigma^{\prime}\right)$ decomposes as a tensor product of quaternion algebras, $(J, \sigma)$ also decomposes as a tensor product of quaternion algebras; in particular, if $\sigma=\operatorname{Int}\left(\sigma^{\prime}(x) x\right) \circ \sigma^{\prime}$, then $(J, \sigma)$ decomposes as $\otimes_{i=1}^{n} \sigma^{\prime}(x) Q_{i} \sigma^{\prime}(x)^{-1}$. Moreover, this decomposition is adapted to $K$ because, by uniqueness of inertial lifts, $\sigma^{\prime}(x) Q_{i} \sigma^{\prime}(x)^{-1}$ contains a lift $K_{i}$ of $\widetilde{K}_{i}$.

In the following theorem, we give necessary and sufficient conditions on when $S$ decomposes into a tensor product of quaternion algebras stable under the involution $\sigma^{*}$. This theorem extends [?, Thm. 4.3] to the case of inertially split division algebras. We will consider the case where $\bar{S}=\bar{Z}$, or, equivalently, when $Z$ is a maximal subfield of $S$; we then only need to work with crossed products and not generalized crossed products. Recall that a crossed product $(Z / F, G, h)$ decomposes into a tensor product of quaternion algebras adapted to $Z$ if and only if $h$ is similar to a symmetric cocycle with values in $F^{*}$ by [?, Cor. 1.4]. We will write $Z^{2}\left(G, F^{*}\right)_{\text {sym }}$ for the group of all symmetric cocycles for $G$ with values in $F^{*}$, and we will use [?, Cor. 1.4] in the proof below. If $A=\otimes_{r=1}^{n}\left(a_{r}, b_{r}\right)$ is a tensor product of quaternion algebras, and if $i_{r}, j_{r} \in\left(a_{r}, b_{r}\right)$ with $i_{r}^{2}=a_{r}, j_{r}^{2}=b_{r}$ and $j_{r} i_{r}=-i_{r} j_{r}$, then we will refer to the set $\left\{i_{1}, j_{1}, \ldots, i_{n}, j_{n}\right\}$ in the proof below as a set of quaternion generators for $A$.

Theorem 4.5 With the notation of this section, suppose further that $\bar{S}=\bar{Z}$. Then the following conditions are equivalent. 
1. The algebra $S$ decomposes into a tensor product of quaternion algebras stable under $\sigma^{*}$;

2. The algebra $S$ has a decomposition adapted to $Z$ as a tensor product of quaternion algebras stable under $\sigma$;

3. The algebra $I$ has a decomposition adapted to $Z$ as a tensor product of quaternion algebras stable under $\sigma$;

4. The algebra $\bar{I}$ has a decomposition adapted to $\bar{Z}$ as a tensor product of quaternion algebras stable under $\bar{\sigma}$.

Proof. $(2) \Rightarrow(1)$ : This is clear.

$(1) \Rightarrow(4)$ : we use the same ideas as in the argument for $(1) \Rightarrow(2)$ of Theorem 4.1. Suppose that $\left\{u_{1}, v_{1}, \ldots, u_{n}, v_{n}\right\}$ forms a set of quaternion generators for $S$ with each $u_{i}$ and $v_{i}$ either symmetric or skew-symmetric with respect to $\sigma^{*}$. We may write $u_{i}=c_{i} y_{g_{i}} w_{i}$ and $v_{i}=d_{i} y_{h_{i}} z_{i}$ with $c_{i}, d_{i} \in Z$ and $w_{i}, z_{i}$ 1-units in $S$ by Lemma 3.1. Moreover, by replacing $u_{i}$ and $v_{i}$ by scalar multiples, we may assume that $c_{i}$ and $d_{i}$ are units since $\Gamma_{Z}=\Gamma_{F}$. A calculation similar to that of the proof of Theorem 4.1 shows that $\left\{\overline{c_{i} x_{g_{i}}}, \overline{d_{i}} \overline{x_{h_{i}}}: 1 \leq i \leq n\right\}$ forms a set of quaternion generators for $\bar{I}$. Since $\bar{S}=\bar{Z}$, we see that $h_{j} \neq h_{i}$ if $j \neq i$, because $\operatorname{Int}\left(\overline{x_{h_{i}}}\right)=h_{i}$ sends $\overline{c_{i} x_{g_{i}}}$ to $-\overline{c_{i} x_{g_{i}}}$ and fixes $\overline{c_{j} x_{g_{j}}}$ if $j \neq i$. Let $a_{i} \in Z$ be a unit that satisfies $h_{i}\left(a_{i}\right)=-a_{i}$ and $h_{j}\left(a_{i}\right)=a_{i}$ if $j \neq i$. Then $\left\{\overline{a_{1}}, \overline{d_{1}} \overline{x_{h_{1}}}, \ldots, \overline{a_{n} x_{h_{n}}}\right\}$ forms a set of quaternion generators for $\bar{I}$, and the corresponding decomposition is adapted to $\bar{Z}$. Furthermore, since $\bar{\sigma}\left(\overline{d_{i}} \overline{x_{h_{i}}}\right)= \pm \overline{d_{i}} \overline{x_{h_{i}}}$, the quaternion factors are each stable under $\bar{\sigma}$.

$(4) \Leftrightarrow(3)$ : This is the second statement of Proposition 4.4 .

$(3) \Rightarrow(2)$. Suppose that $I=(Z / F, G, f)=\oplus_{g \in G} Z x_{g}$ is a tensor product of quaternion algebras each stable under $\sigma$. Then we can write $I=(Z / F, G, e)$ with $e \in Z^{2}\left(G, F^{*}\right)_{\text {sym }}$ by [?, Cor. 1.4]. Moreover, by considering how one writes a tensor product of quaternion algebras as a crossed product, we can write $I=\oplus Z z_{g}$ with $z_{g} z_{h}=e(g, h) z_{g h}$, and each $z_{g}$ is a product of (commuting) elements of a set of quaternion generators of $I$. By the stability under $\sigma$ of these quaternion subalgebras together with the fact that $\left.\sigma\right|_{Z}=$ id, we see that each of the quaternion generators is fixed by $\sigma$. We thus have $\sigma\left(z_{g}\right)=z_{g}$. For the algebra $S$ we have $S=(Z / F, G, e c)$, and since $c \in Z^{2}\left(G, F^{*}\right)_{\text {sym }}$, so also is ec $\in Z^{2}\left(G, F^{*}\right)_{\text {sym }}$. Moreover, if $z_{g}=c_{g} x_{g}$ for some $c_{g} \in Z$, then $g\left(c_{g}\right)=c_{g}$ because $\sigma\left(z_{g}\right)=z_{g}$. Therefore, for $S=(Z / F, G, f c)=\oplus Z y_{g}$, if we set $w_{g}=c_{g} y_{g}$, we have $\sigma^{*}\left(w_{g}\right)=y_{g} c_{g}=g\left(c_{g}\right) y_{g}=w_{g}$ and $w_{g}^{2}=(e c)(g, g) \in F^{*}$. So, $S$ is a tensor product of quaternion algebras each stable under $\sigma^{*}$; if we write $G=\left\langle g_{1}\right\rangle \times \cdots \times\left\langle g_{n}\right\rangle$ and let $Z_{i}$ be the fixed field of $\Pi_{j \neq i}\left\langle g_{j}\right\rangle$, then the quaternion algebras are $Z_{i} \oplus Z_{i} w_{g_{i}}$, which are stable under $\sigma^{*}$ since $\left.\sigma^{*}\right|_{Z}=$ id and $\sigma^{*}\left(w_{g_{i}}\right)=w_{g_{i}}$. This decomposition is clearly adapted to $Z$ since the quaternion algebra $Z_{i} \oplus Z_{i} w_{g_{i}}$ contains the field $Z_{i}$.

In [?, Thm. 4.3] the analogue of condition (2) does not appear. However, the argument given for $(2) \Rightarrow(1)$ in that paper does in fact show that the analogue of this condition is equivalent to the other three conditions of [?, Thm. 4.3]. 


\section{References}

Department of Mathematical Sciences, New Mexico State University, Las Cruces, New Mexico 88003

E-mail address: pmorandi@nmsu.edu

Department of Mathematics, California State Univerisity, Northridge, Northridge, California 91330

E-mail address: al.sethuraman@email.csun.edu 